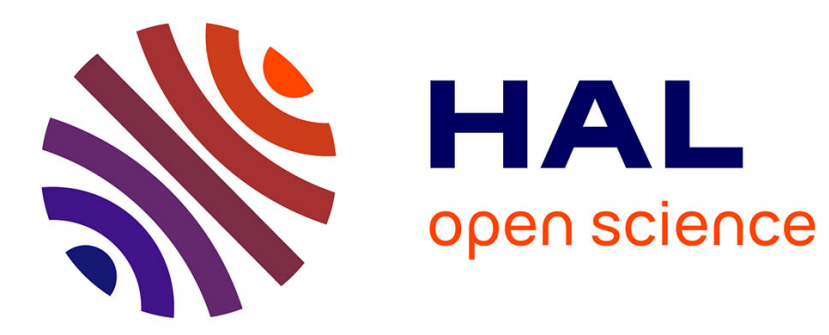

\title{
Managing and recommending resources in web-based collaborative working environments
}

\author{
Siying Li, Marie-Hélène Abel, Elsa Negre
}

\section{To cite this version:}

Siying Li, Marie-Hélène Abel, Elsa Negre. Managing and recommending resources in web-based collaborative working environments. 29th International Conference on Enabling Technologies: Infrastructure for Collaborative Enterprises (WETICE 2020), Sep 2020, Bayonne (virtual), France. pp.287-290.

hal-02944640

\section{HAL Id: hal-02944640 \\ https://hal.science/hal-02944640}

Submitted on 5 Jul 2021

HAL is a multi-disciplinary open access archive for the deposit and dissemination of scientific research documents, whether they are published or not. The documents may come from teaching and research institutions in France or abroad, or from public or private research centers.
L'archive ouverte pluridisciplinaire HAL, est destinée au dépôt et à la diffusion de documents scientifiques de niveau recherche, publiés ou non, émanant des établissements d'enseignement et de recherche français ou étrangers, des laboratoires publics ou privés. 


\section{Managing and recommending resources in web-based collaborative working environments}

\author{
Siying LI \\ Sorbonne universités, Université de \\ technologie de Compiègne \\ CNRS UMR 7253, HEUDIASYC \\ 60203 Compiègne, France \\ siying.li@utc.fr
}

\author{
Marie-Hélène ABEL \\ Sorbonne universités, Université de \\ technologie de Compiègne \\ CNRS UMR 7253, HEUDIASYC \\ 60203 Compiègne, France \\ marie-helene.abel@utc.fr
}

\author{
Elsa NEGRE \\ Paris-Dauphine University, PSL \\ Research University \\ CNRS UMR 7243, LAMSADE \\ 75016 Paris, France \\ elsa.negre@lamsade.dauphine.fr
}

\begin{abstract}
Web-based Collaborative Working Environments intend to support collaborations between users by integrating and offering different collaborative tools. However, this makes it difficult for users to manage and find useful resources to advance their collaborations, which are stored distributively within these tools. This raises an issue: how to manage these resources and identify useful ones for users during their collaborations. In our research, we intend to consider a web-based Collaborative Working Environment as an ontology-based System of Information Systems and to apply a collaboration context ontology that can manage and recommend resources to users within the context of collaboration. In this paper, we present a prototype of such environments and show how context-aware resource recommendations can be generated.
\end{abstract}

Keywords-Collaborative Working Environment, System of Information Systems, Ontology, Collaboration context, Contextaware recommendation.

\section{INTRODUCTION}

With the development of information technology, web-based Collaborative Working Environments (CWEs) [1] can integrate and provide different collaborative tools to users, such as email and real-time chat tools [2] [3]. These tools seek to assist users in their collaborations by producing and/or utilizing diverse resources (e.g. documents and videos). However, these resources are disorganized and stored distributively in distinct tools. This makes it difficult for users to find appropriate and useful resources to carry out their collaborations, which is a barrier to efficient collaboration. Therefore, one of the current issues is how to manage these dispersed resources in a webbased CWE and identify useful ones for users to advance their collaborations.

To solve this issue, it is necessary to understand the relationship between a web-based CWE and its integrated tools where resources are stored. Specifically, each tool can be considered as an information system with its own resource management [4]. These systems, together with the environment itself can form a System of Information Systems (SoIS) [5]. Given the existing resource management in such systems [5], we intend to build a web-based CWE as an ontology-based SoIS. By doing so, dispersed resources can be managed centrally in a shared ontological knowledge base but stored in different information systems.
Besides, these resources are employed during users' collaborations. Accordingly, the utilities of resources are related to collaborations and their contexts (e.g., goals, activities) [6]. This requires us to determine which resources are useful considering the collaboration context, and generate context-aware resource recommendations to users. Hence, we intend to apply a collaboration context ontology as the shared knowledge base in web-based CWEs so that collaboration context can be incorporated while generating resource recommendations.

The reminder of this paper is constructed as follows. Section II studies CWEs, SoISs, and context-aware recommendation. Section III presents our contributions in (i) applying a collaboration context ontology in an architecture of an ontology-based SoIS, (ii) developing a web-based CWE prototype based on the collaboration context ontology and the architecture, (iii) generating context-aware resource recommendations to users. We then discuss the strengths and weaknesses of the prototype and generated recommendations. Finally, some conclusions and future work are put forward in Section V.

\section{RELATED WORK}

This section introduces Collaborative Working Environments and investigates the notion of System of Information Systems. We also explain context-aware recommendations.

\section{A. Collaborative Working Environments}

Nowadays, a new collaborative space, Collaborative Working Environment (CWE), has emerged due to the development of technologies, such as Web/Internet, Information and Communication Technology (ICT), and technologies in the Computer Supported Collaborative Work (CSCW) field [1] [7]. In such environments, users can work together in groups to achieve the common goals of their collaborations [8] [9].

Particularly in web-based CWEs, each group has a space accessible to its members (users) [10]. This permits all members of a group to collaborate in a shared space within the group [8]. Besides, different collaborative tools are also integrated into web-based CWEs [1], including asynchronous ${ }^{1}$ (e.g., email and Wiki) and synchronous ${ }^{2}$ (e.g., real-time chat and

\footnotetext{
${ }^{1}$ Asynchronous collaborative tools let users collaborate at distinct times [2]

${ }^{2}$ Synchronous collaborative tools let users collaborate at the same time [3].
} 
video communication systems) collaborative tools. These tools can produce and/or utilize diverse resources (e.g., documents, figures). This empowers users to collaborate over time and space using different resources [1] [7] [8].

Therefore, many research already developed web-based CWEs for different domains. For example, a CWE was applied to enhance sustainable furniture design [7]. Another CWE was designed for team collaborations [11]. However, there is an unsolved resource burden on users among these existing CWEs: when users shift between groups or perform multiple activities, they must copy and/or move resources between different tools within a CWE [12]. This reflects that resources are stored distributively, either in the tools where they were produced or in the CWE itself, creating difficulties for resource management in a CWE. Without effective resource management, users are incapable of quickly finding appropriate and relevant resources to advance their collaborations in a CWE.

Thus, we need to construct resource management across different tools in a web-based CWE. For this, it is necessary to understand the manner in which collaborative tools are integrated into a CWE. Each tool is an autonomous information system with its own resource management. Together with the CWE, they can form a System of Information Systems (SoIS) [5]. To develop resource management in such an environment, we should also analyze SoIS.

\section{B. Systems of Information Systems}

The notion of System of Information Systems (SoIS) is derived from System of Systems (SoS). A SoS indicates a network of independent and autonomous systems that are integrated together to accomplish common missions [13] [14]. This implies that a SoS consists of two parts: component systems, which are independent and autonomous systems [15], and a global system, which is the collection of its components. As for a web-based CWE, its collaborative tools are component systems, while the CWE itself refers to the global system.

Specifically, a SoIS is a SoS where each component system is an information system [5]. Here, an information system contains a set of interrelated components performing activities for collecting, processing, storing and distributing information [4]. Particularly in a web-based CWE, each collaborative tool can be viewed as an information system with its own resource management. Hence, a web-based CWE is a SoIS. This also indicates that the different resource management in SoISs can also be adapted in web-based CWEs to organize resources.

Notably, an ontology-based SoIS was developed [5], which can manage distributed resources in a shared knowledge base. Considering the relation between web-based CWEs and SoISs, we thus choose to establish a web-based CWE as an ontology-based SoIS so that each resource can be centrally managed in an ontological knowledge base. However, only managing these resources is insufficient. We also need to identify useful resources for users during their collaborations. This necessitates us to consider the utilities of resources within collaboration and its context, and thus generate context-aware resource recommendations. Here, the collaboration context involves any information that can be used to characterize the situation of collaboration over a given period of time [16].

\section{Context-aware recommendation}

To filter irrelevant information and find the most relevant information to users' needs, recommendations are generated by recommender systems (RS) [17]. Usually, two dimensions of data are employed: users and items [18]. Here, items stands for objects that RS recommends to users [19]. When it comes to a web-based CWE, for instance, users can receive resource recommendations to advance their collaborations. In this case, an item is a resource.

Particularly, while generating recommendations, items are identified and sorted by their utilities (ratings) [19]. Such a utility (rating) indicates how a particular user liked a specific item [20]. Given an initial set of ratings that users (explicitly or implicitly) give for items, items' unknown ratings are predicted through utility (rating) functions in RSs [19]. For example, a utility (rating) function of the RSs applying two dimensions of data (written as 2D RS in the following) is [20]:

$$
R_{2 D R S}: \text { User } \times \text { Item } \rightarrow \text { Rating }
$$

where Rating is a totally ordered set (e.g., non-negative integers or real numbers within a certain range).

Recently, a new branch of RS, Context-Aware Recommender System (CARS) [18], was developed. It integrates another dimension of data (i.e., context) with users and items to produce more accurate recommendations, namely contextaware recommendations [21]. Accordingly, the utility (rating) function of CARS is [18]:

$$
R_{\text {CARS }}: \text { User } \times \text { Item } \times \text { Context } \rightarrow \text { Rating }
$$

Here, context is any information that can be used to characterize the situation of users, items, or interactions between users and items [22]. Specifically in our research, context refers to information about users, resources, or the interactions between users and resources in web-based CWEs. All such information can be used to characterize the situation of users' collaborations, and thus belongs to the collaboration context. This suggests that resources should be considered within the collaboration context. Thus, we implement a collaboration context ontology as the knowledge base in web-based CWEs to manage resources with the collaboration context and to generate context-aware resource recommendations.

\section{CONTRIBUTIONS}

This section first shows the resource problems in a collaborative work scenario. To solve such problems, web-based CWEs can be built by applying a collaboration context ontology in the architecture of an ontology-based SoIS. In doing so, we can develop a corresponding prototype of web-based CWE where resources are managed within the collaboration context. Finally, we present the prototype and explain how context-aware resource recommendations can be generated in such environments. 


\section{A. A collaborative work scenario}

Lucie works in a file editor company, FileX. Currently, Lucie is collaborating with her colleagues in two groups to develop two applications of a file editor: a web application and an Android application. Specifically, Lucie, Mary and Steve are working on the web application. Lucie, Emma and Leo collaborate for the Android application. While collaborating, Lucie finds a document ("File editor document 1") and a video ("File editor video") that may be helpful for the development of the two applications. Thus, she wants to share the two resources separately in the two groups and discuss them with other colleagues. However, the two resources are generated and saved in different tools. The video is available on Youtube, while the document is on Lucie's computer. This makes it difficult for Lucie to manage and discuss the two shared resources in two groups. Besides, the desktop application of the file editor was produced by Lucie, Majd and Nathalie.

To let Lucie work more effectively in her company, a webbased CWE can be constructed by applying a collaboration context ontology in the architecture of an ontology-based SoIS. Such a CWE can not only manage resources across different tools, but also generate contextual resource recommendations to Lucie for advancing her collaborations.

\section{B. A collaboration context ontology}

The collaboration context ontology MEMORAeCollaboration-Contect (MCC) [23] intends to describe users' collaborations in groups within web-based CWEs. To represent such collaborations, a concept of user group, mcc:UserGroup (see Fig. 1) is defined and utilized. A mcc:UserGroup contains at least two members (users) that collaborate with each other through their user accounts to achieve their common goals.

Besides, considering a mcc:UserGroup as a subject, all contextual information of collaboration can be detailed by semantic 3-uples $<$ Subject, Predicate, Object $>$ and associated with a subject. In other words, a collaboration and its context in MCC are represented as a collection of semantic 3-uples with a single subject. Particularly, the context of a collaboration is organized in eight dimensions: Goal, Collaborator, Activity, Resource, Time, Location, Relation, and Satisfaction [23]. Each dimension is characterized by one or more predicates and their corresponding objects.

Specifically, all resources are visible and accessible in user groups, regardless of information systems where they are stored. Resources can be deposited either in a web-based CWE (represented by ms:LeaderSystem in Fig. 1), or in a collaborative tool (represented by ms:WebBasedApplication and ms:SandAloneSystem in Fig. 1) integrated into the CWE. Each resource is accessible within the web-based CWE via ms:ReferenceKey and then visible in user groups by mc2:IndexKey (see Fig. 1). When users interacting with resources within user groups, it is the reference and index keys of resources that are employed, rather than themselves stored in different information systems. In Lucie's scenario, the Youtube video applies ms:ReferenceKey to be accessible

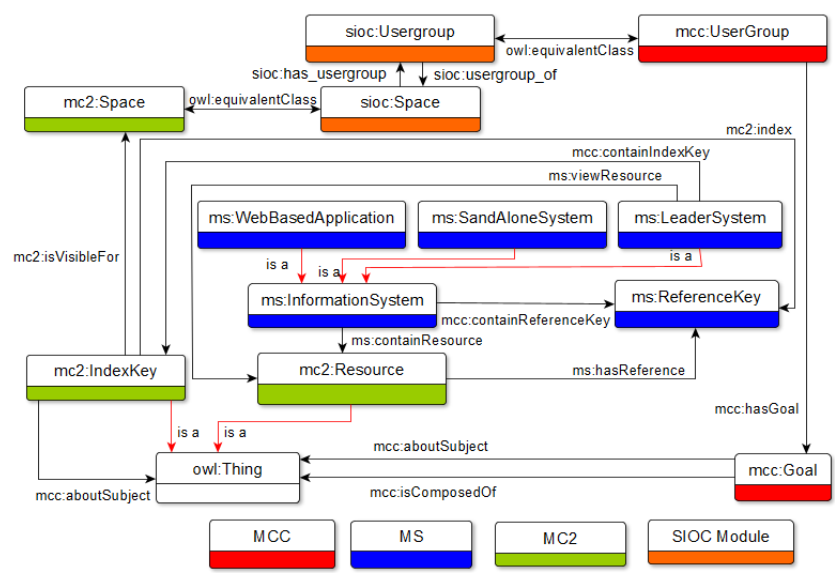

Fig. 1. Resource module in MCC [23].

in web-based CWEs and uses mc2:IndexKey to be visible in Lucie's groups. In addition, each mc2:IndexKey is linked to owl:Thing that refers to a concept in a shared vocabulary. Such a vocabulary aims to describe the goals of collaborations in several relevant user groups. This enables users to index and manage resources across collaborations in a single vocabulary. For example, a vocabulary can be employed for Lucie's two groups to describe their goals so that the Youtube video and the document can be indexed with concepts in the vocabulary and shared in different groups. Thus, through MCC, resources across different systems can be organized and managed within the collaboration context.

Moreover, considering web-based CWEs as ontology-based SoISs, MCC can also serve as an ontological knowledge base in web-based CWEs based on a leader-follower architecture of SoISs [5] (see Part (a) in Fig. 2). In this architecture, a SoIS contains multiple follower systems that are component systems and a leader system that refers to the global system. Adapting the architecture into a web-based CWE (see Part (b) in Fig. 2), the component systems become collaborative tools, while the global system represents the CWE itself.

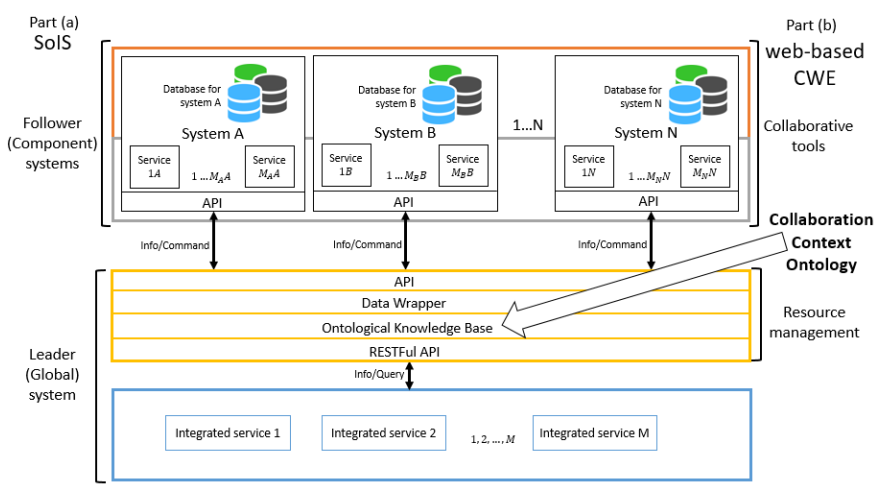

Fig. 2. Considering a CWE as an ontology-based collaborative SoIS.

Particularly, four parts in the CWE aim to centrally manage resources, as follows: API, data wrapper, ontological knowledge base, and RESTFul API. API exchanges information of 
resources with collaborative tools. Then, data wrappers handle and store the collected information in ontological knowledge base. Finally, RESTFul API offers uniform interfaces for accessing and manipulating resources [24]. Such management allows resources located in different tools to be visible and accessible in the CWE. Thus, applying MCC as ontological knowledge base in such an architecture (see Part (b) in Fig. 2) enables us centrally manage dispersed resources within the collaboration context in a web-based CWE. It also makes it possible to develop a corresponding prototype and generate context-aware resource recommendations for users.

\section{Prototype}

By applying the collaboration context ontology in the architecture of an ontology-based SoIS (cf. Section III-B), we construct a web-based CWE prototype: MEMORAe CWE.

In MEMORAe CWE, users collaborate in user groups. In Lucie's scenario, the two groups for developing different applications are two user groups in MEMORAe CWE. Particularly, each user group owns a space that enables its members to access and interact with resources stored distributively within MEMORAe CWE. For example, Lucie can share a web link to the Youtube video in the group space of the web application and index it with the concept File editor in a shared vocabulary (see Fig. 3). Here, the web link is a resource that is stored in Youtube but accessible in MEMORAe CWE via ms:ReferenceKey. It is as well visible and indexed within a user group via mc2:IndexKey. Apart from web link, a resource can also be a document, an annotation, a vote, a comment, or a geographical location, stored either in integrated tools or in MEMORAe CWE itself. All integrated tools can be found in the pop-up window Add a resource (see Fig. 3).

Several relevant user groups can form an organization in MEMORAe CWE. Each organization has a shared vocabulary visualized as a knowledge graph to describe goals of collaborations. In the graph, each node symbolizes a concept in the vocabulary. In the scenario, Lucie's two user groups form an organization. The goals of the two user groups can thus be described in a knowledge graph of this organization (see Fig. 3). All members of the two user groups can consult the graph in MEMORAe CWE.

Particularly, the nodes in knowledge graphs serve to index resources in user groups. Users need to select the indexed node to access and view resources within user groups. In the scenario, the web link shared by Lucie is indexed with the node File editor (see Fig. 3). When selecting this particular node in the knowledge graph, Lucie can access and view the web link in the corresponding group space. Meanwhile, Lucie can also open other group spaces from a list to consult other indexed resources (see Fig. 3). Notably, resources are visible and accessible only with the indexed nodes. This indicates that in the group space of the web application, Lucie can not access or view the web link while selecting other nodes.

Besides, all resources in MEMORAe CWE are managed within user groups through semantic 3-uples $<$ Subject, Predicate, Object $>$ in the collaboration context ontology. Specif- ically, considering a user group as a subject, resources are objects related to this subject. In the collaboration context ontology, there is no limit to the number of objects related to a subject. Thus, users in MEMORAe CWE can index any number of resources within a user group. Together, these resources constitute a set of objects related to a single subject. With such sets of objects, we can compare the similarity of resources between collaborations within user groups, and thus to identify useful resources based on user groups. In other words, this enables us to generate context-aware resource recommendations for users in MEMORAe CWE.

\section{Context-aware resource recommendation}

Considering a user group as a collection of semantic 3-uples in the collaboration context ontology, the indexed resources in a user group $c$ is a set of objects $O^{c}$. In Lucie's scenario, if the user group of the web application is $c$ and Lucie only index resources with the node File editor in $c$, then we can obtain $O^{c}=\{$ "File editor video" $\}$. Thus, the context-aware resource recommendation problem ${ }^{3}$ is: given a user $u$ in a user group $c$ with a set of indexed resources $O^{c}$ and a utility (rating) matrix $R$, the top $K$ resources $i\left(i \notin O^{c}\right)$ that can be indexed in the user group $c$ with the highest probabilities will be recommended to $u$ for facilitating $u$ 's collaboration in the user group $c$. Here, the context includes $u$ 's collaboration in the user group $c$ and the set of resources $O^{c}$.

Addressing this recommendation problem requires us to incorporate the context into the recommendation generation process. To this end, we first predict resources' unknown ratings $r^{*}$ through the rating function $R_{2 D} R S$ (see Equation $1)^{4}$ to complete the rating matrix $R$. Next, we calculate user $u$ 's adjusted ratings $\widehat{r}$ depending on the user $u$ 's ratings in $R$ and the context. Finally, the top $K$ resources with $K$ highest adjusted ratings $\widehat{r}$ will be recommended to the user $u$. By doing so, the context is integrated into resource recommendations, thereby changing the rating function $R_{2 D} R S$ (see Equation 1) into $R_{C A R S}$ (see Equation 2$)^{5}$.

Specifically, while applying the context in generating recommendations, we employ a semantic similarity of resources between collaborations within user groups. To calculate such a similarity, two sets of resources are compared in terms of number of resources and number of common resources. Thus, based on [25] [26], the semantic similarity of resources between two user groups ( $c$ and $y$ ) is ${ }^{6}$ :

$$
S(c, y)=\frac{\left|O^{c} \cap O^{y}\right| \times W \operatorname{eight}\left(p^{c, \text { Res }}\right)}{\left|O^{c} \cap O^{y}\right|+\alpha\left|O^{c}-O^{y}\right|+\beta\left|O^{y}-O^{c}\right|}
$$

where $O^{c}$ is a set of all resources indexed within the user group $c ; O^{c}-O^{y}$ is the relative complement of $O^{y}$ in $O^{c}$; $\left|O^{c} \cap O^{y}\right|$ is the number of common resources indexed within

\footnotetext{
${ }^{3}$ The utility (rating) matrix $R$ of $M \times N$ contains users' known ratings for resources ( $M$ users and $N$ resources).

${ }^{4}$ In our research, Item in Equation 1 refers to Resource.

${ }^{5}$ In our research, Item in Equation 2 refers to Resource.

${ }^{6} W$ eight $\left(p^{c, R e s}\right), \alpha$ and $\beta$ are calculated or given based on the semantic 3 -uples. We do not discuss how they are calculated or given in this paper.
} 


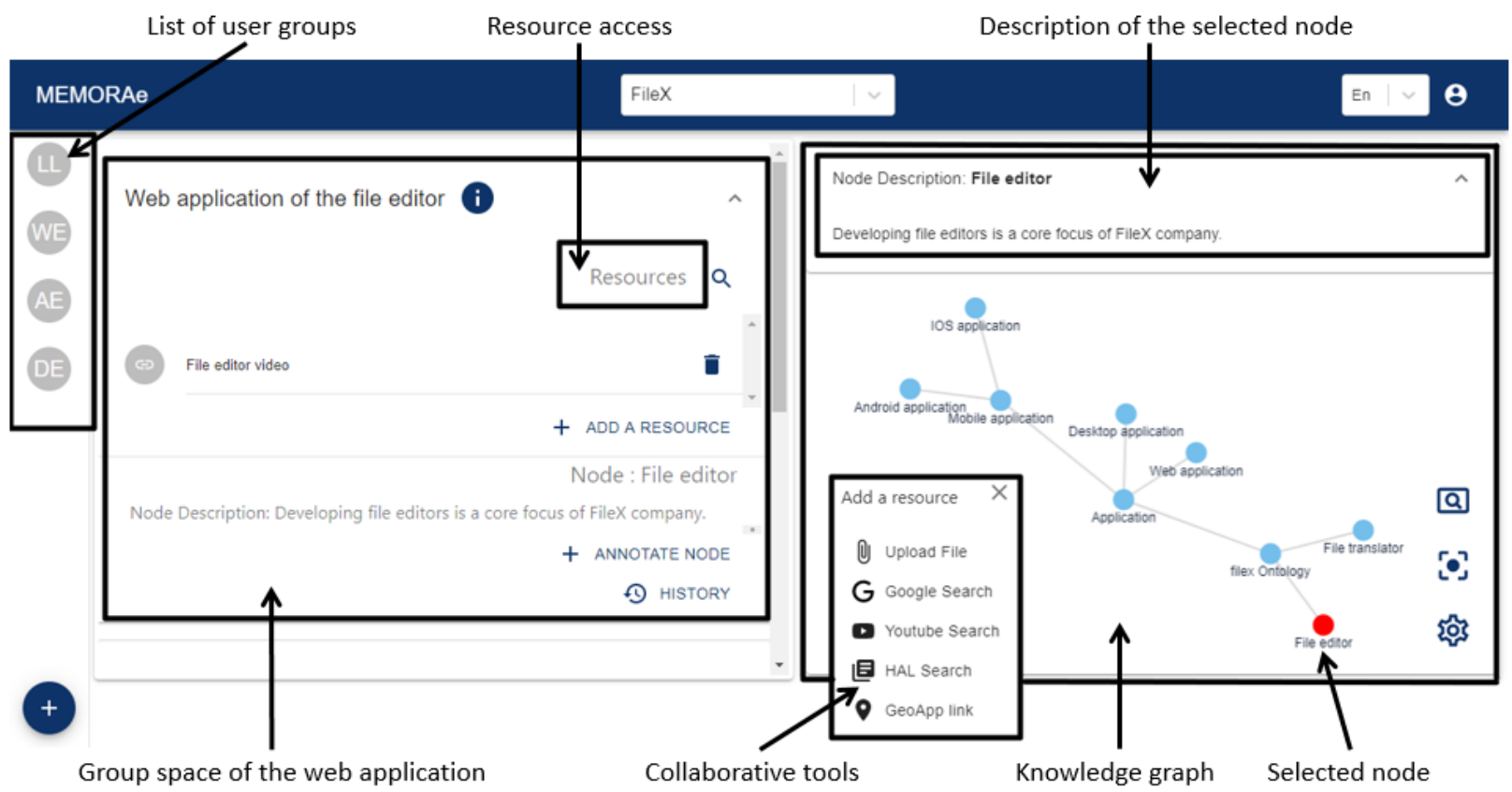

Fig. 3. Lucie's interface in MEMORAe CWE.

user groups $c$ and $y$; Weight $\left(p^{c, \text { Res }}\right)$ is the significance of resources in the user group $c ; \alpha, \beta(\geq 0)$ refer respectively to the weights of user groups $c$ and $y$.

Then, the user $u$ 's adjusted rating for the user group $y$ is $\widehat{r}(u, y)=w_{\text {similarity }} * S(c, y)+w_{\text {rating }} * r(u, y)$. Here, $w_{\text {similarity }}, w_{\text {rating }}(\geq 0)$ refer to the weights of $S(c, y)$ and $u$ 's rating for $y(r(u, y) \in R)$. Accordingly, the algorithm to generate context-aware resource recommendations ${ }^{7}$ is presented in Algorithm 1.

In the scenario, we consider $K=1$ and $u=$ Lucie to illustrate how a resource can be recommended to Lucie. Thus, the user group of the web application is $c$; $t_{1}$ and $t_{2}$ are user groups of the Android application and the desktop. Then, we have $O^{c}=\{"$ File editor video" $\}, O^{t_{1}}=$ $\{$ "File editor document 1", "Annotation" $\}$, and $\mathrm{O}^{t_{2}}=$ \{"File editor video", "File editor document 1", "Note" \}.

Following the algorithm, we first need to predict $\mathrm{Lu}$ cie's unknown ratings for resources. In this paper, we do not discuss how to predict unknown ratings. Therefore, Lucie's ratings for resources are provided as follows: $r$ (Lucie,"File editor document 1") $=0.8$, $r($ Lucie, "Annotation" $)=0.6$, and $r($ Lucie,, Note" $)=$ 0.2 . Then we can calculate $S\left(c, t_{1}\right), S\left(c, t_{2}\right)$. Suppose that $W$ eight $\left(p^{c, \text { Res }}\right)=\alpha=\beta=1$, we can get $S\left(c, t_{1}\right)=$ $\frac{0 \times 1}{0+1 \times 1+1 \times 2}=0$ and $S\left(c, t_{2}\right)=\frac{1 \times 1}{1+1 \times 0+1 \times 2}=\frac{1}{3} \approx 0.333$. $S\left(c, t_{2}\right)>S\left(c, t_{1}\right)$ indicates that $t_{2}$ has more common resources to $c$ than $t_{1}$.

Next, we can compute adjusted ratings $\widehat{r}$ based on Lucie's ratings and semantic similarities. Suppose that $w_{\text {similarity }}=$

\footnotetext{
${ }^{7}$ In this algorithm, the ranges of ratings $r_{i j}$ and semantic similarities $S(c, t)$ are same. We do not discuss how to adapt the range of semantic similarities into the range of ratings in this paper.
}

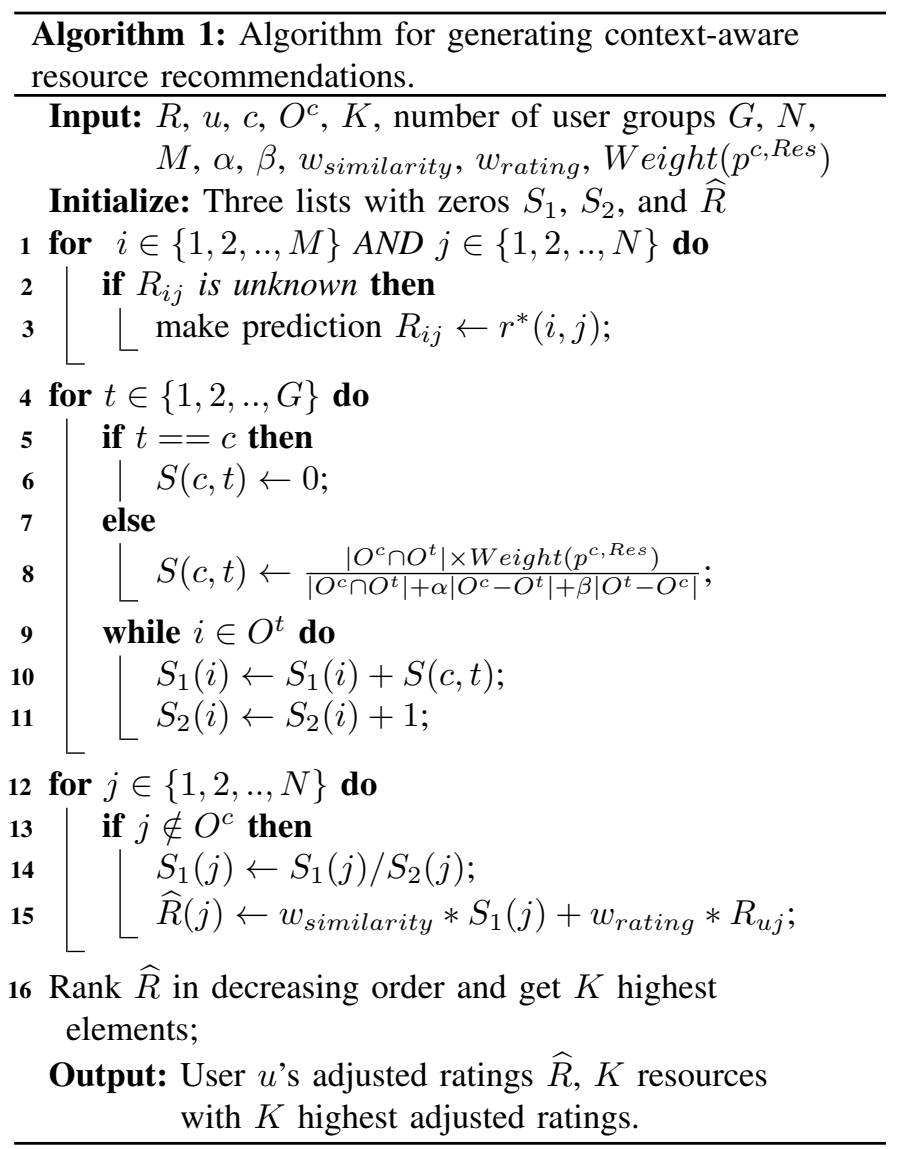


$w_{\text {rating }}=1$, then $\widehat{r}($ Lucie,, File editor document $1 ")=$ $\frac{0+0.333}{2} \times 1+0.8 \times 1=0.9665, \widehat{r}($ Lucie,, Annotation" $)=$ $\frac{0}{1} \times 1+0.6 \times 1=0.6$, and $\widehat{r}($ Lucie, "Note" $)=$ $\frac{0.333}{1} \times 1+0.2 \times 1=0.533$. This indicates that $\widehat{r}$ (Lucie, "File editor document 1") is Lucie's highest adjusted rating for resources. Thus, "File editor document 1 " should be recommended to Lucie. Within the collaboration context ontology, resource recommendations can be generated by considering the collaboration context in user groups.

\section{Discussion}

Considering a web-based Collaborative Work Environment (CWE) as an ontology-based information system (SoIS) creates an opportunity to construct centralized resource management through separate tools within a CWE. Such management also enables users to index and organize resources within user groups, thus solving users' resource burden [12] in existing CWEs. Besides, applying the collaboration context ontology [23] in web-based CWEs permits us to consider and identify the utilities of resources within the collaboration context. This, in turn, helps to generate more accurate resource recommendations for users in web-based CWEs, context-aware resource recommendations [21]. Particularly, such recommendations collectively consider users and items (resources) through using the collaboration context, rather than considering user or item (resource) context separately.

\section{CONCLUSION AND FUTURE WORK}

In this paper, we focus on how to manage resources in a web-based Collaborative Working Environment (CWE) and identify useful ones for users. To solve this issue, we develop a web-based CWE prototype by applying a collaboration context ontology into the architecture of an ontology-based System of Information Systems (SoIS) and generate context-aware resource recommendations for users. Based on the literature review, we justified the relation between web-based CWE and SoIS, and explained how context-aware recommendations can be generated. We investigated how a collaboration context ontology can be adapted into a web-based CWE via the architecture of an ontology-based SoIS and utilized in generating context-aware recommendations. The corresponding prototype and recommendation algorithm were then presented. Finally, their characteristics were discussed.

Our future work includes testing the recommendation algorithm and developing a corresponding recommender system as a new tool integrated into web-based CWEs.

\section{REFERENCES}

[1] M. A. Martínez-Carreras, A. Ruiz-Martínez, F. Gomez-Skarmeta, and W. Prinz, "Designing a generic collaborative working environment," in IEEE International Conference on Web Services (ICWS 2007), 2007, pp. $1080-1087$.

[2] J. Xu, J. Zhang, T. Harvey, and J. Young, "A survey of asynchronous collaboration tools," Information Technology Journal, vol. 7, no. 8, pp. 1182-1187, 2008.

[3] N. Wang, "Towards a competency recommender system from collaborative traces," Ph.D. dissertation, Université de Technologie de Compiègne, 2016.
[4] V. V. G. Neto, R. Araujo, and R. P. dos Santos, "New challenges in the social web: Towards systems-of-information systems ecosystems," in Anais do VIII Workshop sobre Aspectos da Interação HumanoComputador para a Web Social. SBC, 2017, pp. 1-12.

[5] M. Saleh and M.-H. Abel, "Moving from digital ecosystem to system of information systems," in 2016 IEEE 20th International Conference on Computer Supported Cooperative Work in Design (CSCWD). IEEE, 2016, pp. 91-96.

[6] H. Patel, M. Pettitt, and J. R. Wilson, "Factors of collaborative working: A framework for a collaboration model," Applied ergonomics, vol. 43, no. 1, pp. 1-26, 2012.

[7] D. Su and J. Casamayor, "Web-based collaborative working environment and sustainable furniture design," in Proceedings of the 19th CIRP Design Conference-Competitive Design, 2009.

[8] G. Bafoutsou and G. Mentzas, "Review and functional classification of collaborative systems," International journal of information management, vol. 22, no. 4, pp. 281-305, 2002.

[9] I. Oliveira, L. Tinoca, and A. Pereira, "Online group work patterns: How to promote a successful collaboration," Computers \& Education, vol. 57, no. 1, pp. 1348-1357, 2011.

[10] R. Bentley, W. Appelt, U. Busbach, E. Hinrichs, D. Kerr, K. Sikkel, J. Trevor, and G. Woetzel, "Basic support for cooperative work on the world wide web," International journal of human-computer studies, vol. 46, no. 6, pp. 827-846, 1997.

[11] H.-L. Truong, S. Dustdar, D. Baggio, S. Corlosquet, C. Dorn, G. Giuliani, R. Gombotz, Y. Hong, P. Kendal, C. Melchiorre et al., "Incontext: A pervasive and collaborative working environment for emerging team forms," in 2008 International Symposium on Applications and the Internet. IEEE, 2008, pp. 118-125.

[12] G. H. ter Hofte, "Working apart together: Foundations for component groupware," pp. 34-52, 1998.

[13] M. Jamshidi, Systems of systems engineering: principles and applications. CRC press, 2008.

[14] N. Karcanias and A. G. Hessami, "System of systems and emergence part 1: Principles and framework," in 2011 Fourth International Conference on Emerging Trends in Engineering \& Technology, pp. 27-32.

[15] M. A. ASSAAD, R. Talj, and A. Charara, "A view on systems of systems (sos)," in 20th World Congress of the International Federation of Automatic Control (IFAC WC 2017) - special session, 2016.

[16] S. Li, M.-H. Abel, and E. Negre, "Contact and collaboration context model," in 2018 IEEE 4th International Forum on Research and Technology for Society and Industry (RTSI). IEEE, 2018, pp. 1-6.

[17] I. Nunes and D. Jannach, "A systematic review and taxonomy of explanations in decision support and recommender systems," User Modeling and User-Adapted Interaction, vol. 27, no. 3-5, pp. 393-444, 2017.

[18] G. Adomavicius, R. Sankaranarayanan, S. Sen, and A. Tuzhilin, "Incorporating contextual information in recommender systems using a multidimensional approach," ACM Transactions on Information Systems (TOIS), vol. 23, no. 1, pp. 103-145, 2005.

[19] F. Ricci, L. Rokach, and B. Shapira, "Introduction to recommender systems handbook," in Recommender systems handbook, 2011, pp. 1-35.

[20] G. Adomavicius and A. Tuzhilin, "Toward the next generation of recommender systems: A survey of the state-of-the-art and possible extensions," IEEE Transactions on Knowledge \& Data Engineering, no. 6, pp. 734-749, 2005.

[21] C. Palmisano, A. Tuzhilin, and M. Gorgoglione, "Using context to improve predictive modeling of customers in personalization applications," IEEE transactions on knowledge and data engineering, vol. 20, no. 11, pp. $1535-1549,2008$

[22] A. K. Dey, "Understanding and using context," Personal and ubiquitous computing, vol. 5, no. 1, pp. 4-7, 2001.

[23] S. Li, M.-H. Abel, and E. Negre, "Towards a collaboration context ontology," in 2019 IEEE 23rd International Conference on Computer Supported Cooperative Work in Design (CSCWD), 2019, pp. 93-98.

[24] R. Lucchi, M. Millot, and C. Elfers, "Resource oriented architecture and rest," Assessment of impact and advantages on INSPIRE, Ispra: European Communities, 2008.

[25] W. Carrer-Neto, M. L. Hernández-Alcaraz, R. Valencia-García, and F. García-Sánchez, "Social knowledge-based recommender system. application to the movies domain," Expert Systems with applications, vol. 39, no. 12, pp. 10990-11000, 2012.

[26] J. Rahnama and E. Hüllermeier, "Learning tversky similarity," in International Conference on Information Processing and Management of Uncertainty in Knowledge-Based Systems. Springer, 2020, pp. 269-280. 


\section{APPENDIX}

This paper is a modified version of submission <paper $25>$ in the previous round. Accordingly, the title of the paper has been changed from A Collaborative Working Environment as an ontology-based collaborative System of Information Systems to Managing and recommending resources in webbased collaborative working environments for adaptation to the modifications.

Here are our responses and modifications to the comments received by the reviewers in the previous round.

- REVIEW 1: The proposal is an interesting solution and it deserves to be presented and discussed in the Conference. The creation of a web-based CWE by adapting the architecture of an ontology-based collaborative SoIS and applying the collaboration context ontology to this objective is appreciated as a useful contribution to improving the resources organization.

At the same time, this approach can improve the integration of different collaborative tools in an effective way, while the contextual information of collaborations allows users to adapt themselves to better collaborate among them.

It is shown a very interesting and formalized analysis of requirements that supports the design and development of the prototype MEMORAe CWE.

RESPONSE: Thank you very much for your comments and remarks. We have concentrated more on resource organization and management in web-based CWEs in the modified version by explaining the algorithm of context-aware resource recommendations. At the same time, we have also applied a collaborative work scenario to demonstrate the prototype MEMORAe CWE.

- REVIEW 2: The article summaries the MEMORAe CWE platform. The contribution section seems to be a summary of the platform in term of architecture (Collaborative Working Environment), Collaboration context ontology[9], and prototype. what is the originality of this specific article ? S. Li, M.-H. Abel, and E. Negre, "Towards a collaboration context ontology," in 2019 IEEE 23rd International Conference on Computer Supported Cooperative Work in Design (CSCWD). IEEE, 2019, pp.93-98.

The resource recommendation problem is not introduced theoretically in previous sections. There are new way to calculate similarity between two resource sets in user groups according to user personal interest or personal information. [19] and [20] are old references. [19] A. Tversky, "Features of similarity." Psychological review, vol. 84, no. 4, p. 327, 1977. [20] W. CarrerNeto, M. L.Hern'andez-Alcaraz, R. Valencia-Garc'sa, and F. Garc'1a-S'anchez, "Social knowledge-based recommender system. application to the movies domain," Expert Systems with applications, vol. 39, no. 12, pp. 10 990-11 000, 2012.

There is no qualitative or quantitative performance eval- uation of the efficiency of the recommendation algorithm and similarity according to the end-user expectations. reference [10] has a strange author format. [10] "Contact and collaboration context model," in 2018 IEEE 4th International Forum on Research and Technology for Society and Industry (RTSI). IEEE, 2018, pp. 1-6.

RESPONSE: Thank you very much for your comments and remarks. To better explain the originality of this paper, we have reformulated our research problem in the modified version and represented it through a collaborative work scenario. Besides, we have also theoretically introduced recommendations and context-aware recommendation in Section II-C, explained the recommendation algorithm by the scenario and pseudo-code, and added a recent reference (i.e. [26]) on similarities. As for qualitative or quantitative performance evaluation of the recommendation algorithm, our recommendation algorithm are going to be tested as our future work. Finally, we have checked and corrected the author format of references by changing the default setting in the file IEEEtran.bst.

- REVIEW 3: The paper is well written and easy to read but you have not achieved your goals. They were ambitious and propose three things (i) adapting an SoIS Platform ...; (ii) applying a context ontology and (iii) developing a collaborative working environment. What is the problem? It seemed that it is an upgrade of an already existed system but it is not entirely clear.

The platform exists, the ontology too. Maybe the example of sharing a text tool using both Android and computer platforms is the test. In that case, the use of knowledge graphs and the context should be emphasized, and results must be showed. In order to improve your paper you need to briefly describe the platform, the ontology and fully describe you experiment, the goals, and the achievements. RESPONSE: Thank you very much for your comments and remarks. To better explain the problem in this paper, we have reformulated our research problem and presented it through a collaborative work scenario. We have also described how to solve the research problem by presenting the platform, the ontology and the recommendation algorithm within the scenario. 